INPLASY

PROTOCOL

To cite: Wang. Efficacy and safety of different IL-6 inhibitors in COVID-19: a network meta-analysis. Inplasy protocol 202140007. doi: 10.37766/inplasy2021.4.0007

Received: 01 April 2021

Published: 02 April 2021

Corresponding author: Zhenhu Wang

1416889858@qq.com

Author Affiliation:

Beijing University of traditional Chinese Medicine

Support: No financial support.

Review Stage at time of this submission: Formal screening of search results against eligibility criteria.

Conflicts of interest: None declared.

\section{Efficacy and safety of different IL-6 inhibitors in COVID-19: a network meta-analysis}

Wang, $Z^{1}$.

Review question / Objective: This study intends to evaluate the efficacy and safety of different IL-6 inhibitors in the treatment of COVID-19 by network meta-analysis, in order to explore the best IL-6 inhibitor therapeutic drugs. P: COVID-19 patients, I: different IL- 6 inhibitors, C: the control group can be different from the intervention group IL-6 inhibitors, can also be standard care and hormone and other conventional treatment, O: COVID-19 patients with treatment response rate, mortality, and complications.

Condition being studied: At present, many studies have reported the efficacy of different IL-6 inhibitors in the treatment of COVID-19, but different IL-6 inhibitors are reported in the literature, and the efficacy is controversial. Network meta-analysis is an effective method, which can be indirectly compared to obtain which IL-6 inhibitor is the best therapeutic drug. Existing conditions include the database for obtaining original literatures and mature statistical method for network meta-analysis.

INPLASY registration number: This protocol was registered with the International Platform of Registered Systematic Review and Meta-Analysis Protocols (INPLASY) on 02 April 2021 and was last updated on 02 April 2021 (registration number INPLASY202140007).

\section{INTRODUCTION}

Review question / Objective: This study intends to evaluate the efficacy and safety of different IL- 6 inhibitors in the treatment of COVID-19 by network meta-analysis, in order to explore the best IL-6 inhibitor therapeutic drugs. P: COVID-19 patients, I: different IL-6 inhibitors, C: the control group can be different from the intervention group IL-6 inhibitors, can also be standard care and hormone and other conventional treatment, O: COVID-19 patients with 
treatment response rate, mortality, and complications.

Rationale: There are a variety of drugs for the treatment of COVID-19 with IL-6 inhibitors, such as Ruxolitinib, Tocilizumab, Baricitinib, and Sarilumab, and the therapeutic effects of various drugs are different and controversial, and the current COVID-19 epidemic is still more serious, and it is meaningful to select effective drugs to treat patients by means of network meta-analysis.

Condition being studied: At present, many studies have reported the efficacy of different IL- 6 inhibitors in the treatment of COVID-19, but different IL-6 inhibitors are reported in the literature, and the efficacy is controversial. Network meta-analysis is an effective method, which can be indirectly compared to obtain which IL-6 inhibitor is the best therapeutic drug. Existing conditions include the database for obtaining original literatures and mature statistical method for network metaanalysis.

\section{METHODS}

Search strategy: Term: IL-6 inhibitors, Ruxolitinib, Tocilizumab, Baricitinib, and Sarilumab, COVID-19. The databases searched included: pubmed, embased, Cochrane Libirary, China National Knowledge Infrastructure (CNKI), Wanfang database, etc.

\section{Participant or population: COVID-19} Patients.

Intervention: Different IL-6 inhibitors, such as IL-6 inhibitors, Ruxolitinib, Tocilizumab, Baricitinib, and Sarilumab.

Comparator: IL-6 inhibitors or standardized nursing and routine western medicine treatment are different from those in the intervention group.

Study designs to be included: Randomized Controlled Trials and Cohort Studies.
Eligibility criteria: 1, the study subjects in the literature were COVID-19 patients. 2, the interventions were different IL-6 inhibitors. 3. The study type is randomized controlled trial or cohort study.

Information sources: The databases searched included: pubmed, embased, Cochrane Libirary, China National Knowledge Infrastructure (CNKI), Wanfang database, etc.

Main outcome(s): The effective rate, mortality and complication rate of treatment.

Additional outcome(s): Changes of serum inflammatory factors, such as C-reactive protein content, white blood cell count, lymphocyte count, etc.

Data management: At first, the two researchers screened the retrieved literature independently according to the inclusion and exclusion criteria, then crosschecked, and the controversial literature was evaluated by the third party and then unified through discussion.

Quality assessment / Risk of bias analysis: The included studies were randomized controlled studies or cohort studies. The Jadada scale was used to assess the quality of randomized controlled studies, and the scores $(0 \sim 3)$ were classified as low quality articles and $(4 \sim 7)$ as high quality articles. The Newcastle-Ottawa Scale (NOS) form was used for quality assessment of the cohort study, with scores of $(0 \sim 4)$ classified as low quality articles and $(5 \sim 9)$ as high quality articles.

Strategy of data synthesis: The data were analyzed using STATA version 16.0 software. Enumeration data were expressed as relative risk (RR), measurement data were expressed as weighted mean standard deviation (WMD), and interval estimation was performed using $95 \%$ confidence interval $(\mathrm{Cl})$ as an indicator of effect size. When bringing the data extracted from the literatures into the Stata16.0 software for operation, the direct comparison result is compared with the 
indirect comparison result by using the node-splittingmodel in the software to observe whether the two results are consistent and then clarify the consistency test result. If there was no statistical difference ( $P>0.05)$, the consistent model was used for network meta-analysis of the efficacy of various drug treatments.

Subgroup analysis: Since it is a network meta-analysis, we no longer perform the analysis.

Sensitivity analysis: Since it is a network meta-analysis, we will not perform the sensitivity analysis.

Language: Language is restricted to Chinese and English.

Country(ies) involved: China.

Other relevant information: No.

Keywords: COVID-19, IL-6 inhibitor, network meta-analysis, efficacy, complications.

Contributions of each author:

Author 1 - Zhenhu Wang. 